\title{
Die Reserven der Krankenkassen
}

\author{
Jürg Baumberger
}

Jeweils im Herbst, wenn uns die Prämienerhöhungen wie ein jährlich wiederkehrendes Naturereignis ins Haus flattern, kommen mit schöner Regelmässigkeit auch die Reserven der Krankenversicherungen ins Gespräch. Die auf der hohen Kante liegenden Milliarden wecken Gelüste, seien diese finanzieller, seien diese politischer Art. Die zumindest teilweise Auflösung der Reserven könnte doch, so der naheliegende Gedanke, die Prämienlast etwas dämpfen.

1 Wacker P. A propos des hausses annoncées de cotisations par les caisses-maladie. Schweiz. Ärztezeitung 2005;86(41):2319

Korrespondenz:

Dr. phil. Jürg Baumberger

Health Management Institute

HMI creative AG

Im Hauweg 6

CH-8370 Sirnach

E-Mail: jb@juergbaumberger.ch

Internet: www.juergbaumberger.ch
Die Versicherungen scheinen reich zu sein mit ihren an «Übergewicht leidenden Reserven» («leurs réserves frappées d'embonpoint») [1]. Aber die Sache ist so einfach nicht. Die goldene Reservekuh, die zugunsten der Prämien geschlachtet werden könnte, ist eine Mär, alt und anscheinend unausrottbar.

\section{Gewinnverbot}

Gemäss Artikel 13 KVG dürfen die Krankenversicherer ihre Mittel nur zugunsten der sozialen Krankenversicherung verwenden. Sie dürfen also keinen Gewinn machen. Das zeigen auch die im KVG für die Versicherer vorgesehenen Rechtsformen. Sie dürfen aber auch nicht bankrott gehen, denn sie haben sicherzustellen, dass sie ihren finanziellen Verpflichtungen jederzeit nachkommen können. Deshalb schreibt Art. 60 KVG vor, dass die Finanzierung selbsttragend zu sein hat.

\section{Reserven und Rückstellungen}

Zur Absicherung unvorhergesehener Entwicklungen und daraus folgender Kosten müssen die Versicherer eine Sicherheitsreserve bilden. Damit sichern sie ab, dass sie die Rechnungen der Partner zahlen können, auch bei unvorhergesehenen Ereignissen wie Epidemien. Art. 78 KVV schreibt vor, dass die Reserve für Versicherer bis 250000 Versicherte 20 Prozent einer Jahreseinnahme an Prämien sein muss, für grössere Versicherer beträgt sie 15 Prozent.

Die Versicherer haben Rückstellungen für ausstehende Versicherungskosten zu bilden. Diese decken die Rechnungen, die nach Ablauf des Prämienjahres noch eintreffen. Diese kommen auch noch für Versicherte herein, die nicht mehr bei der Versicherung sind. Und sie kämen auch, wenn die Versicherung ihr Geschäft auf Jahresende einstellen würde. Die Höhe dieser Rückstellungen richtet sich nach Erfahrungszahlen der vorangegangenen Jahre.

Sowohl Reserven als auch Rückstellungen sind abhängig von der Kostensteigerung im Gesundheitswesen, die Reserven von der für die Zahlung von Rechnungen erforderlichen Prämienhöhe, die Rückstellung vom Rechnungseingang.

\section{Gesetzliche Auflagen nur teilweise erfüllt}

Wie sehen nun die Zahlen aus? Gemäss Statistik des Bundesamtes für Gesundheit BAG betrugen 1996 die Reserven aller Versicherer 25,7 Prozent, 2004 waren es noch 16,5 Prozent, nachdem sie 2002 auf 12,8 Prozent abgesackt waren. Das sind Durchschnittszahlen.

Im einzelnen sieht das nach der Statistik des Bundesamtes für Statistik BfS so aus, dass 2002 von den 10 Versicherern mit über 250000 Versicherten eine über 20 Prozent Reserven hatte, 5 zwischen 15 und 17 Prozent und 4 unter 15 Prozent. Eine Versicherung hatte sogar negative Reserven. Bei den 10 nächstkleineren Versicherern hatten nur 2 die erforderliche Quote von 20 Prozent, 5 waren unter 15 Prozent, 1 negativ.

Die Rückstellungen sanken seit der Einführung des KVG von durchschnittlich 32 Prozent einer Jahresausgabe auf 27,5 Prozent im Jahre 2004. Anscheinend kommen heute die Rechnungen schneller herein.

Dass die Zinsen und Renditen von Reserven und Rückstellungen als neutrale Erträge die Prämienlast etwas dämpfen, wird oft vergessen. Die anders als in Hochzinszeiten zurzeit etwas sparsamer fliessenden Mittel aus diesen Quellen leisten trotz allem einen regelmässigen Beitrag.

\section{Statt behoben nur verschoben}

Die politisch dekretierte (Dreifuss) und nun wieder diskutierte (Couchepin) Variante, die vorgeschriebene Reservehöhe abzusenken, ist problematisch. In dem Jahr, in dem die Versicherung Reserven auflöst, macht sie technisch Verlust. 
Sie nimmt weniger Prämien ein, als sie Ausgaben zu decken hat. Das ist das Gegenteil von nachhaltig, denn die Probleme werden nicht nur in die Zukunft verschoben, sondern auch noch verschärft. Wenn die Möglichkeit der Reserveauflösung nicht mehr gegeben ist, muss bei der jährlichen Prämienberechnung zuerst die alte Defizitlücke geschlossen und dann erst noch die $\mathrm{zu}$ erwartende Kostensteigerung eingerechnet werden. Ein doppelter Prämiensprung also. Und wenn auf der Kostenseite keine grundsätzliche Besserung eintritt, sind wir in kurzer Zeit wieder da, wo wir heute stehen.
Die von Bundesrat Couchepin geforderte Senkung von in welschen Kantonen über dem gesetzlichen Minimum liegenden Reserven ist zwar politisch einleuchtend, aber auch hier gilt die Regel des anstehenden doppelten Prämienbedarfs.

Dass die gleichen Probleme sich auch einer Einheitskasse stellen, die mit Kopfprämien finanziert würde, sei am Rande vermerkt. Es sei denn, diese hätte keine Reserven zu bilden und der Staat würde quasi als Rückversicherer auftreten. Aber damit wäre das oben beschriebene Problem auch hier statt behoben nur verschoben. 